\title{
Acute Necrotizing Pancreatitis with Gastric Necrosis in a Female with Cerebral Palsy
}

\section{Kurdi $\mathbf{M}^{*}$ and Shkrum M}

Department of Pathology and Laboratory Medicine, Western University, London Ontario, Canada

${ }^{*}$ Corresponding author: Kurdi M, MD, FRCPC. EFN, Department of Pathology and Laboratory Medicine, London Health Sciences Centre, 339 Windermere Rd London, Ontario, Canada N6A 5A5, Fax: 519-6632930, Tel: 519-685-8500,E-mail: Drc_marmar@hotmail.com

Citation: Kurdi M, Shkrum M (2016) Acute Necrotizing Pancreatitis with Gastric Necrosis in a Female with Cerebral Palsy. J Case Rep Stud 4(4): 402. doi: 10.15744/2348-9820.4.402

Received Date: May 15, 2016 Accepted Date: August 27, 2016 Published Date: August 30, 2016

\begin{abstract}
The coexistence of drug -induced pancreatitis and gastric necrosis is very rare. Many drugs have been associated with pancreatitis and among the antiepileptic drugs; valproic acid has been commonly implicated. Carbamazepine-induced pancreatitis has been rarely reported in the literature because linking this medication to pancreatitis has been difficult given the wide range of more common etiologies. We report an autopsy case of acute pancreatitis with gastric necrosis in a 27 -year-old female with cerebral palsy on long term carbamazepine therapy. The causal association of acute pancreatitis and gastric necrosis is discussed based on review of the literature.
\end{abstract}

Keywords: Pancreatitis; Drug induced pancreatitis; Carbamazepine

\section{Case Presentation}

A 27-year-old woman with history of cerebral palsy and epilepsy was transferred from a community hospital to a tertiary care hospital with hypovolemic shock. The patient had a two-hour history of abdominal discomfort and distension. She was on longterm carbamazepine therapy because of epilepsy. The family administered her medication and did not indicate any history of excess medication ingestion. Laboratory investigations showed a metabolic acidosis, elevated pancreatic enzymes: amylase up to $1906 \mathrm{u} / \mathrm{L}$ (normal range $28-100 \mathrm{u} / \mathrm{L}$ ); lipase $3126 \mathrm{u} / \mathrm{L}$ on admission at the peripheral hospital rising to $7611 \mathrm{u} / \mathrm{L}$ the next day (normal range 13-60 u/L) and coagulopathy. A CT scan of the abdomen revealed gaseous gastric dilatation and pancreatic enhancement, consistent with pancreatitis. Subsequent gastroesophageal endoscopy revealed no esophageal abnormalities. There was an extensive necrosis involving the entire stomach (Figure 1). There was no evidence of perforation.

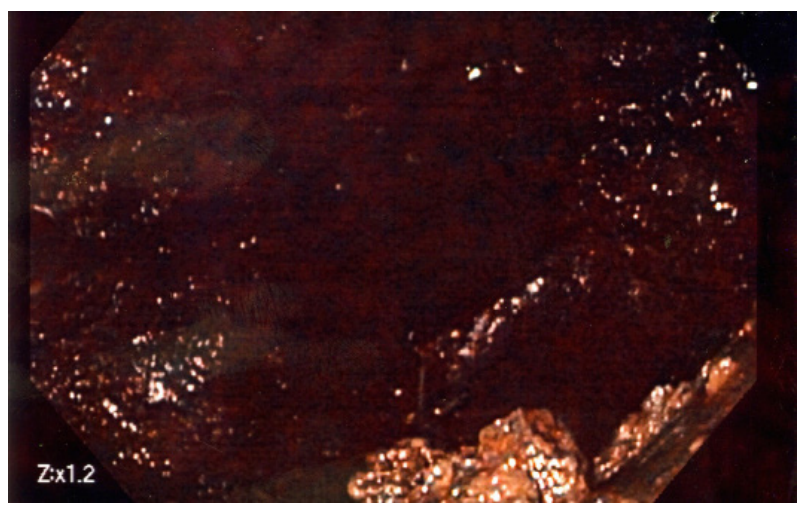

Figure 1: Gastroesophageal endoscopy shows severe gastric necrosis

Because the side effects of carbamazepine therapy were not clinically suspected, a carbamazepine level was not measured during admission. On the second day, the serum carbamazepine level (25 micromoles/L) was within the normal therapeutic range (17-51 micromoles/L). She was placed on comfort measures. The autopsy was limited to the abdominal and pelvic cavities. It revealed pancreatic and perigastric fat necrosis (Figure 2). There was massive gastric dilatation with extensive necrosis (Figure 3). Microscopic examination showed foci of acute fat and pancreatic necrosis (Figure 4) and confirmed acute transmural gastric necrosis (Figures 5). There was no evidence of vasculitis. 


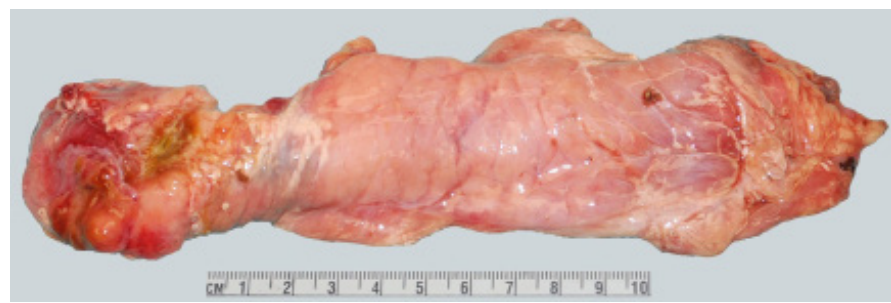

Figure 2: Autopsy gross imaging shows acute pancreatitis and fat necrosis

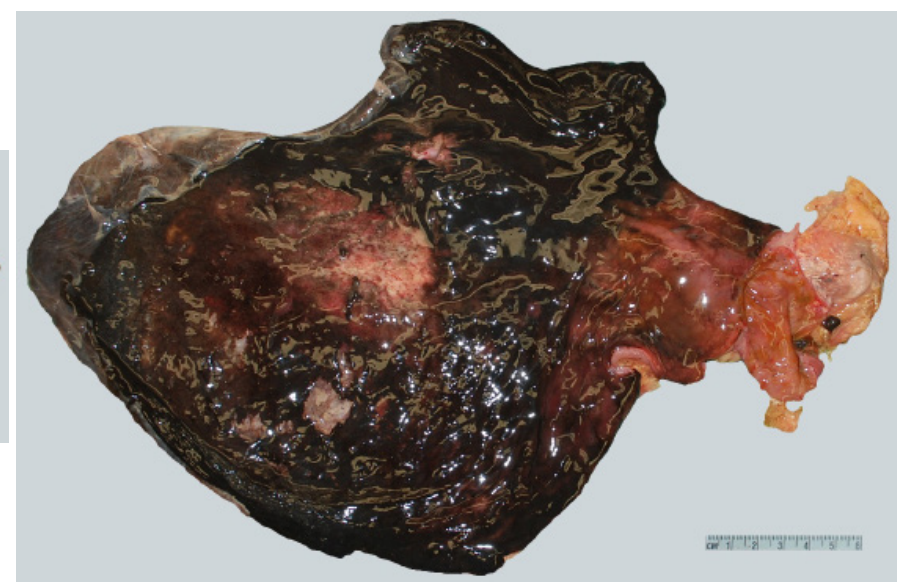

Figure 3: Autopsy gross imaging shows diffuse gastric necrosis

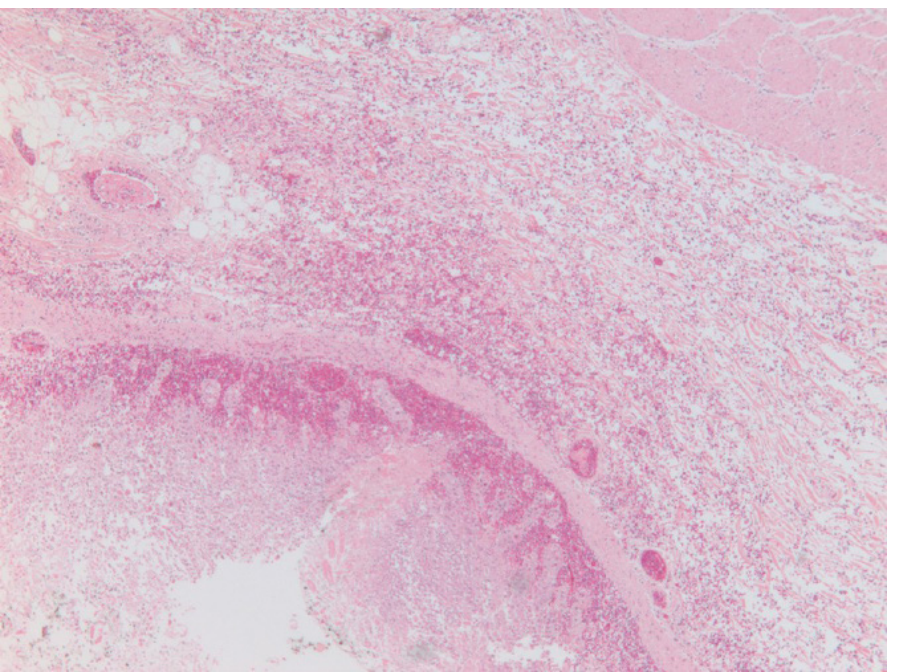

Figure 5: Histology section (H\&EX50) shows transmural gastric necrosis

Figure 4: Histology section (H\&EX100) shows pancreatic necrosis

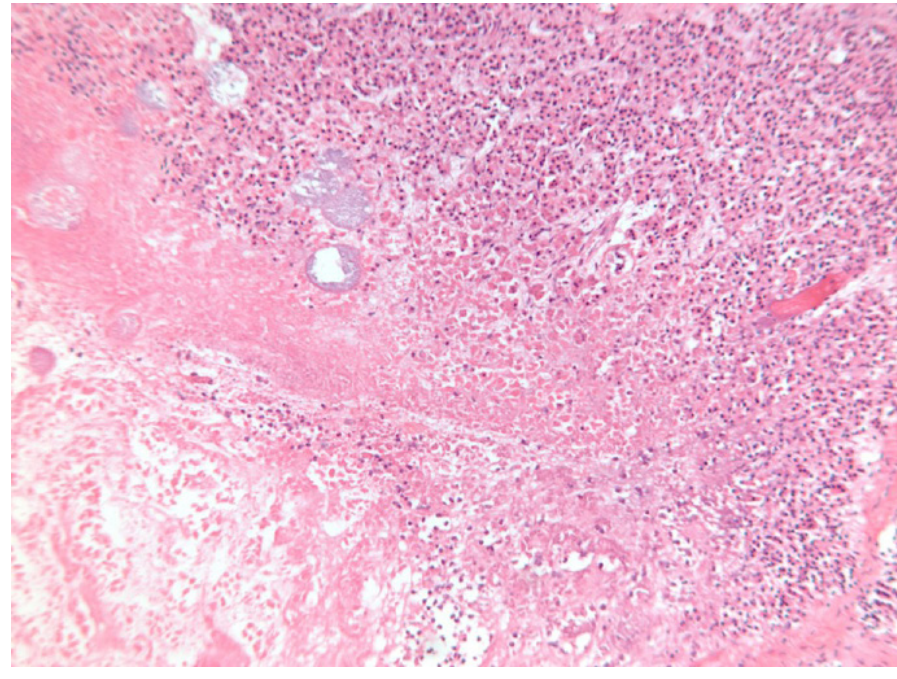

\section{Discussion}

The most common causes of acute pancreatitis are cholelithiasis and alcoholism which were factors not found in our case. Other causes of pancreatitis such as hemolytic-uremic syndrome, sepsis, viral infection, hypercholesterolemia, systemic lupus erythematosus, cystic fibrosis, and corticosteroid use did not appear to be present in our case. Because of the short clinical course, assessment of some of these factors, such as infection, could not be done. The plasma urea level was $4.6 \mathrm{mmol} / \mathrm{L}$ (normal $<8.3$ $\mathrm{mmol} / \mathrm{L})$.

Hereditary pancreatitis (HP) is an autosomal dominant disease characterized by chronic pancreatitis during childhood without the identification of known inciting factors. There is no consensus about the diagnostic criteria for HP, but the EUROPAC trial defined it as two first-degree relatives or at least three second-degree relatives in two or more generations [1]. In our case, there was no history of hereditary pancreatitis in the family.

Autoimmune pancreatitis is a form of chronic pancreatitis of presumed autoimmune etiology. The disease is recognised as a Tcell-mediated specific disease with lymphoplasmatic infiltration of pancreatic tissue and pancreatic parenchyma fibrosis. Serum immunoglobulin IgG or IgG4 and antibodies are usually increased [2]. Our patient was not tested for these markers. There was no evidence of lymphoplasmatic inflammation or fibrosis in the histology sections.

If all the causes of pancreatitis have been excluded, then idiopathic pancreatitis needs to be considered in the differential diagnosis. Patient characteristics that are associated with a pancreas-specific genetic mutation include a personal and family history of chronic pancreatitis or the presence of pancreas divisum. Patients with CFTR, PSTI or N34S gene mutations are at much higher risk to develop chronic pancreatitis [3]. The role of specific genetic aberrations associated with chronic pancreatitis raises the question whether genetic testing should be routinely performed or only when idiopathic chronic pancreatitis is suspected. Whether a specific gene mutation in synergy with carbamazepine therapy predisposed this patient to develop pancreatitis is uncertain.

Drug-induced or -associated pancreatitis is rare and is described only in case reports. The diagnosis of drug-induced pancreatitis is likely underestimated because of the difficulties in linking it to medication use. Many drugs have been associated with pancreatitis 
[4]. Venkilerova, et al. [5] concluded in their study, on 170 cases with pancreatitis, that $5.3 \%$ of acute pancreatitis cases were due to drugs, and it was the third most frequent cause of the pancreatitis. Azathioprine was the most frequent drug in their series. Among the antiepileptic drugs, valproate was the first reported to be associated with acute pancreatitis $[5,6]$. There are no clinical criteria or specific diagnostic tests, which assist in establishing the definitive diagnosis of drug-induced pancreatitis. Serum drug levels must be measured on admission to determine whether toxic concentrations may be present. This is because metabolism of the medication will lower its concentration during the subsequent clinical course. In the present case, the level of carbamazepine was in the therapeutic range, but this was determined the day after admission Carbamazepine, a class III drug, has rarely been reported to be associated with pancreatitis [4,7-11]. It has calcium and sodium antagonistic properties and it may cause hypocalcemia [12]. We did not find any evidence in the literature that carbamazepine-induced hypocalcemia can cause pancreatitis. The actual mechanism associating carbamazepine and pancreatitis has not been discussed in the literature due to small number of proven cases.

The patient in the present case report had gastric necrosis and dilatation, likely consequences from acute pancreatitis [13]. Gastric ischemia and necrosis can develop due to the impairment of the intramural blood flow from gastric dilatation [14]. Microcirculatory effects of inflammatory mediators decrease blood flow to stomach and have been proven to play a role in development of gastric necrosis [13].

A few cases of acute pancreatitis causing gastric necrosis have been reported in the literature (Table 1). The pathophysiological mechanism of this association is not fully understood. Individuals with acute pancreatitis can develop vascular complications such as thrombosis of peripancreatic and perigastric veins or coeliac artery trunk $[13,15,16]$. Vascular complications in this case were not identified.

\begin{tabular}{|c|c|c|c|c|}
\hline Case & Authors & Age & Sex & Underlying disease or factors \\
\hline 1 & Kumaran et al. [16] & 58 & M & Coeliac artery trunk thrombosis \\
\hline 2 & Scholefield et al. [15] & 48 & M & Visceral ischemia \\
\hline 3 & Hsu et al. $[17]$ & 40 & M & Pancreatic pseudocyst \\
\hline 4 & Rieger et al. $[13]$ & 50 & M & Alcoholism \\
\hline 5 & Scheppach et al. [18] & 60 & M & Cholesterol embolism \\
\hline 6 & Umeda et al. [19] & 49 & M & Aortic dissection \\
\hline
\end{tabular}

Table 1: Few Cases of Acute Pancreatitis causing Gastric Necrosis

\section{Conclusion}

Drug-induced pancreatitis may be under-recognized as a significant contributor to patient morbidity and mortality. It can be complicated by gastric necrosis. The diagnosis of drug-induced pancreatitis is underestimated because of the difficulties in determining a drug as the causative agent by means of any specific diagnostic tests. Based on literature review, drug-induced pancreatitis is more probable in younger persons and patients suffering inflammatory bowel diseases such as Crohn's disease or epilepsy. Carbamazepine use is rarely complicated by pancreatitis. The mechanism is unknown due to the small number of reported cases. Regular monitoring of serum amylase and lipase should be considered in patients who are on long-term antiepileptic drug therapy.

\section{References}

1. Lal A, Lal DR (2010) Hereditary pancreatitis. Pediatr Surg Int 26: 1193-9.

2. Dite P, Novotony I, Tna J, Sevcikova A (2008) Autoimmune pancreatitis. Best Pract Res Clin Gastroenterol 22: 131-43.

3. DiMagno EP (2001) Gene mutation and idiopathic chronic pancreatitis: clinical implication and testing. Gastroenterology 121: 1508-12.

4. Mallory A, Kern F (1980) Drug-induced pancreatitis: a critical review. Gastroenterology 78: 813-20.

5. Vinklerová I, Procházka M, Procházka V, Urbánek K (2010) Incidence, severity, and etiology of drug-induced acute pancreatitis. Dig Dis Sci 55: $2977-81$.

6. Batalden PB, van Dyne BJ, Cloyd J (1979) Pancreatitis associated with Valporic acid therapy. Pediatrics 64: 520-2.

7. Soman M, Swenson C (1985) A possible case of carbamazepine induced pancreatitis. Drug Intell Clin Pharm 19: 925-7.

8. Tsao CY, Wright FS (1993) Wright, Acute chemical pancreatitis associated with carbamazepine intoxication. Epilepsia 34: 174-6.

9. Arshad F, Ladakhi Y, Khan MA (2013) A rare case of carbamazepine induced pancreatitis. Int J Stud Res 3: 59.

10. Laczek JT, Shrestha M, Kortan ND, Lake JM (2010) Carbamazepine-induced pancreatitis with positive rechallenge. J Clin Gastroenterol 44: 153-4.

11. Forte A, Gallinaro L, Montesano G, Turano R, Bertagni A, et al. (1996) A possible case of carbamazepine induced pancreatitis. Riv Eur Sci Med Farmacol 18: 187-9.

12. Ambrósio AF, Soares-Da-Silva P, Carvalho CM, Carvalho AP (2002) Mechanisms of action of carbamazepine and its derivatives, oxcarbazepine, BIA2-093 and BIA2-024. Neurochem Res 27: 121-30.

13. Rieger A, Bachmann J, Schulte-Frohlinde E, Burzin M, Nährig J, et al. (2012) Total gastric necrosis subsequent to acute pancreatitis. Pancreas 41: 325-7.

14. Jefferiss CD (1972) Spontaneous rupture of the stomach in an adult. Br J Surg 59: 79-80. 
15. Scholefield JH, Goodman AJ, Morgan WP (1988) Abdominal wall and gastric infarction in acute pancreatitis. Pancreas 3: 494-6.

16. Kumaran C, Chung AF, Ooi LP, Chow PH, Wong WK (2006) Coeliac artery trunk thrombosis in acute pancreatitis causing total gastric necrosis. ANZ J Surg 76: $273-4$.

17. Hsu CY, Lee KC, Chan CC, Lee FY, Lin HC (2009) Gastric necrosis and perforation as a severe complication of pancreatic pseudocyst. J Chin Med Assoc 72: 603-6.

18. Scheppach W, Teschner M, Kirchner T, Schindler C, Franke S, et al. (1993) Acute pancreatitis and stomach wall necrosis caused by cholesterol embolisms. Dtsch Med Wochenschr 118: 13-8.

19. Umeda I, Hayashi T, Ishiwatari H, Yoshida M, Miyanishi K, et al. (2011) A case of sever acute pancreatitis and ischemic gastropathy caused by acut aortic dissection. Nihon Shokakiyo Gakki Zasshi 108: 103-10. 